\title{
Efeito de um programa educativo no conhecimento e na qualidade dos registros da pressão arterial*
}

\author{
Ana Carolina Queiroz Godoy Daniel ${ }^{1}$ \\ (D) https://orcid.org/0000-0003-4877-9191 \\ Eugenia Velludo Veiga ${ }^{2}$ \\ (i) https://orcid.org/0000-0003-3677-0210 \\ Juliana Pereira Machado ${ }^{3}$ \\ (1D) https://orcid.org/0000-0003-2225-8355 \\ Ana Carolina Cintra Nunes Mafra ${ }^{4}$ \\ (D) https://orcid.org/0000-0001-9004-7176 \\ Lyne Cloutier ${ }^{5}$ \\ (D) https://orcid.org/0000-0003-2886-6538
}

\footnotetext{
* Artigo extraído da tese de doutorado "Programa educativo sobre registro da pressão arterial em serviço hospitalar de emergência: um estudo de intervenção", apresentada à Universidade de São Paulo, Escola de Enfermagem de Ribeirão Preto, Centro Colaborador da OPAS/OMS para o Desenvolvimento da Pesquisa em Enfermagem, Ribeirão Preto, SP, Brasil.

${ }^{1}$ Hospital Israelita Albert Einstein, Unidade de Pronto Atendimento, São Paulo, SP, Brasil.

2 Universidade de São Paulo, Escola de Enfermagem de Ribeirão Preto, Centro Colaborador da OPAS/OMS para o Desenvolvimento da Pesquisa em Enfermagem, Ribeirão Preto, SP, Brasil.

${ }^{3}$ Centro Universitário Barão de Mauá, Ribeirão Preto, SP, Brasil.

${ }^{4}$ Hospital Israelita Albert Einstein, São Paulo, SP, Brasil.

${ }^{5}$ Université du Québec à Trois-Rivières, Département de Sciences Infirmières, Trois-Rivières, QC, Canadá.
}

Objetivo: avaliar o efeito de um programa educativo sobre o registro da pressão arterial para profissionais de enfermagem em relação ao conhecimento teórico e à qualidade desses registros. Método: estudo quase-experimental conduzido em um serviço hospitalar localizado no município de São Paulo. O conhecimento teórico de 101 profissionais foi mensurado por meio de questionário validado antes e após a intervenção educativa. A qualidade dos registros da pressão arterial foi avaliada utilizando-se formulário validado e aplicado a 354 prontuários no período pré-intervenção e 288 no período pós-intervenção. O programa educativo foi fundamentado em metodologias ativas de ensino-aprendizagem e constituído por duas estratégias: aula expositiva-dialogada e um jogo de tabuleiro. Os testes de Wilcoxon, Mann-Whitney, Fisher e Qui-Quadrado foram utilizados para comparações, adotandose um nível de significância de $a=0,05$. Resultados: a mediana de acertos dos profissionais passou de 19 para 22 pontos no período pós-intervenção $(p<0,001)$. Houve melhora na qualidade dos registros de pressão arterial quanto às variáveis tamanho do manguito $(p<0,001)$, membro utilizado no procedimento $(p<0,001)$ e posição do paciente $(p<0,001)$. Conclusão: o programa educativo mostrou resultados positivos na promoção do conhecimento dos profissionais de enfermagem e na melhora da qualidade dos registros da pressão arterial.

Descritores: Registros Médicos; Determinação da Pressão Arterial; Educação Profissionalizante; Educação Continuada; Educação em Enfermagem; Materiais de Ensino.

\section{Como citar este artigo}

Daniel ACQG, Veiga EV, Machado JP, Mafra ACCN, Cloutier L. Effect of an educational program for the knowledge and quality of blood pressure recording. Rev. Latino-Am. Enfermagem. 2019;27:e3179. [Access Available in: DOI: http://dx.doi.org/10.1590/1518-8345.3011.3179. 


\section{Introdução}

O registro da pressão arterial (PA) é uma das etapas que compõem o procedimento de medida indireta da PA, o qual fornece informações importantes para o cuidado assistencial e fundamenta a avaliação clínica, a definição diagnóstica e as condutas terapêuticas de pacientes acometidos pelas mais diversas condições de saúde ${ }^{(1-2)}$. Em termos gerais, esse registro pode possibilitar a troca de informações entre os membros da equipe multiprofissional, o contato com o histórico de saúde do paciente e a avaliação da qualidade da assistência prestada(3).

Diretrizes nacionais e internacionais de hipertensão arterial (HA) recomendam que o registro da PA seja composto pela documentação da posição do corpo do paciente, do membro utilizado para a colocação do manguito e dos valores de pressão arterial sistólica (PAS) e pressão arterial diastólica (PAD) obtidos imediatamente após a realização do procedimento(1-2).

Apesar do significado que o registro da PA traz para o cuidado do paciente, principalmente no que diz respeito à definição diagnóstica e ao planejamento de tratamentos e intervenções, estudos demonstraram que as condutas dos profissionais de enfermagem frente à documentação dos valores de PA ainda são defasadas e não retratam os cuidados recebidos pelo cliente no momento da assistência à saúde ${ }^{(4-5)}$. Além disso, o conhecimento teórico e prático sobre o procedimento de medida indireta da PA está aquém do esperado para o cumprimento correto da técnica(6-7)

Intervenções educativas baseadas em metodologias ativas de ensino têm sido aplicadas aos estudantes de enfermagem, demonstrando resultados positivos no aprendizado da técnica de medida indireta da $\mathrm{PA}^{(8-9)}$. Entretanto, a utilização dessas estratégias tem sido questionada devido à falta de embasamento do método pedagógico e ao consequente comprometimento da qualidade das evidências produzidas sobre a formação profissional em saúde ${ }^{(10)}$.

No ambiente organizacional, as atividades lúdicas têm se destacado por facilitar a construção do conhecimento, melhorar o desempenho dos profissionais de saúde em suas atividades cotidianas e promover a adesão às diretrizes e aos protocolos estabelecidos na prática clínica(11). A incorporação de jogos educativos, nesse contexto, visa favorecer o aprendizado de conceitos e técnicas, a visão crítica, o encorajamento profissional, a interação social, a criatividade, a imaginação, a cognição e a emoção(12).

Muito embora o registro da PA tenha sido reconhecido como etapa do procedimento de medida indireta da PA há mais de trinta anos, sua importância para a qualidade do processo assistencial tem sido pouco discutida nos meios científico e acadêmico.
Nesse sentido, o desenvolvimento de pesquisas e a implementação de estratégias educativas voltadas para esse registro podem contribuir com a prática baseada em evidências, com a qualidade do cuidado e a segurança dos pacientes atendidos nos diferentes níveis de atenção à saúde.

Diante do exposto, o objetivo do presente estudo foi avaliar o efeito de um programa educativo sobre o registro da PA para profissionais de enfermagem em relação ao conhecimento teórico e à qualidade desses registros. Um objetivo secundário foi comparar duas abordagens educativas: aula expositiva-dialogada com aula expositivadialogada somada à aplicação de um jogo de tabuleiro.

\section{Método}

Trata-se de um estudo com delineamento quase-experimental, conduzido entre dezembro de 2015 e junho de 2016, em um serviço hospitalar de emergência de grande porte localizado no município de São Paulo. Os estudos quase-experimentais são considerados como de intervenção e diferenciam-se dos experimentais verdadeiros por não contemplarem o processo de randomização ou mesmo a definição de um grupo controle(13).

O modelo quase-experimental do tipo antes e depois com amostras independentes ${ }^{(14)}$ foi utilizado para avaliar a qualidade dos registros de PA nos períodos pré e pós-intervenção. Já o modelo antes e depois com um grupo(14) comparou o conhecimento teórico dos profissionais de enfermagem antes e após a aplicação do programa educativo.

Para detectar as diferenças médias relevantes na qualidade dos registros de PA entre os dois períodos, seriam necessários, ao menos, 88 observações em cada um, ao se considerar testes de comparação de proporções, tamanhos de efeito de 0,3, com nível de significância de $5 \%$ e poder de teste de $80 \%^{(15)}$. A amostra de prontuários foi composta pelo total de atendimentos realizados na unidade de emergência, nos meses de dezembro de 2015 e junho de 2016. Foram excluídos prontuários de pacientes com abalos emocionais e/ou agitação psicomotora, que poderiam dificultar a medida e o registro periódico da PA. As condições físicas e psíquicas de tais pacientes foram identificadas por meio dos registros realizados pela equipe médica e de enfermagem durante o período de atendimento no referido serviço de saúde.

Para comparar o conhecimento teórico antes e após a implementação do programa educativo, todos os profissionais de enfermagem foram convidados a participar da intervenção, realizada entre os meses de janeiro a maio de 2016. O cálculo do tamanho da 
amostra revelou que seria necessário um mínimo de 90 participantes para conduzir um teste t pareado, capaz de comparar as pontuações obtidas no questionário aplicado a cada profissional, antes e após o período de intervenção, considerando-se um nível de significância de $5 \%$, tamanho de efeito de 0,3 e poder de teste de $80 \%{ }^{(15)}$. Tendo em vista a intenção dos pesquisadores em disponibilizar o programa educativo para toda a equipe assistencial, a amostra foi composta pelo quadro total de trabalhadores elegíveis, o que resultou em um número maior do que o planejado.

Foram excluídos os profissionais que estavam em afastamento do trabalho, os que se recusaram a participar da pesquisa e aqueles que não completaram todas as etapas do estudo: assinatura de termos de consentimento, preenchimento de questionários ou participação na intervenção educativa.

A qualidade dos registros da PA foi avaliada pelo formulário intitulado "Qualidade dos Registros da Pressão Arterial em Prontuário" (QRPAP), enquanto o conhecimento teórico dos profissionais de enfermagem foi avaliado pelo questionário denominado "Conhecimento Teórico sobre Registro da Pressão Arterial em Serviço Hospitalar de Emergência" (CTRPA-SHE). Ambos os instrumentos foram desenvolvidos no ano de 2015 por pesquisador do estudo e validados quanto à aparência e ao conteúdo por um grupo de seis especialistas, os quais foram elencados a partir dos seguintes critérios: ser enfermeiro; com titulação de mestre ou doutor; ter experiência profissional na área assistencial; ter conhecimento metodológico sobre a construção de questionários e escalas; e atuar na área de ensino e pesquisa com temática voltada a saúde cardiovascular, medida da pressão arterial, hipertensão arterial, segurança do paciente, inovação educacional ou assuntos correlatos.

O QRPAP foi construído com base em documentos da literatura que versavam sobre a medida e o registro da $P A^{(1-2)}$, sendo sua versão final constituída por cinco itens de registros: da medida da circunferência braquial (CB), do tamanho do manguito utilizado, do membro em que foi medida a $C B$, da posição do paciente e dos valores de PA com a unidade de medida do aparelho em milímetros de mercúrio $(\mathrm{mmHg})$.

O CTRPA-SHE foi desenvolvido a partir de uma vasta revisão da literatura sobre registros de enfermagem e medida da $\mathrm{PA}^{(7,16-18)}$, sendo composto por seis itens referentes à identificação do participante (data de nascimento, sexo, categoria profissional, tempo de formação profissional, tempo em que trabalha na instituição de saúde e turno de trabalho) e mais 23 itens distribuídos em oito questões de múltipla escolha, com quatro opções de resposta, e três questões do tipo verdadeiro e falso, compostas por cinco subitens. Ao total, o CTRPA-SHE foi formado por 11 questões que abordaram os seguintes temas: importância do registro da PA para o cuidado de enfermagem, registro dos valores de PA nos casos de instabilidade hemodinâmica, registro dos valores alterados de PA, registro da PA com aparelhos automáticos e suas vantagens, registro da posição do paciente, registro da frequência cardíaca, registro do tamanho do manguito, registro da PA conforme rotina hospitalar, frequência de registro da PA conforme quadro clínico do paciente, frequência de registro da PA conforme prescrição de enfermagem e registro das etapas que compõem o procedimento de medida da PA.

O questionário CTRPA-SHE foi submetido à validação semântica pelo método DISABKIDS ${ }^{\circledR(19)} \mathrm{com}$ a finalidade de verificar se todos os itens do instrumento de coleta de dados são compreensíveis para todos os membros da população focalizada. Uma amostra de conveniência constituída por três profissionais de enfermagem de diferentes níveis de formação foi elencada para atingir todos os estratos da população do estudo e garantir a fidedignidade das respostas durante o processo de validação.

A técnica Delphi(20) foi o método utilizado para sistematizar o consenso dos especialistas quanto ao julgamento dos itens do formulário QRPAP e do questionário CTRPA-SHE. O Índice de Validade de Conteúdo (IVC) ${ }^{(21)}$ foi aplicado para verificar o grau de concordância dos especialistas em cada item dos instrumentos, sendo considerados válidos aqueles que obtiveram IVC maior ou igual a $80 \%$.

A intervenção baseou-se na implementação de um programa educativo intitulado "Pressão e Ação", desenvolvido por um dos autores deste estudo e fundamentado em documentos elaborados pelas sociedades de especialistas em $\mathrm{HA}^{(1,22-23)}$. A aula expositiva-dialogada e o jogo de tabuleiro foram as estratégias de ensino utilizadas no período de intervenção, ambos pautados no conceito de metodologias ativas de ensino-aprendizagem sob a perspectiva da autonomia da educação(24-25).

Para a aula expositiva-dialogada, foram elaborados cartazes e crachás ilustrados que continham orientações sobre o registro da medida indireta da PA em impresso institucional específico, com campos destinados à documentação do tamanho do manguito, do membro utilizado no procedimento, do decúbito do paciente, da frequência cardíaca e dos valores de PA em $\mathrm{mmHg}$.

O jogo de tabuleiro foi composto por 50 cartas de perguntas e respostas, cinco peões coloridos, um dado, um manual de instruções e um tabuleiro dividido em cinco eixos temáticos: fisiopatologia da $\mathrm{HA}$, conceitos da $\mathrm{PA}$, aparelhos oscilométricos de PA, manguitos de PA, procedimento de medida da PA e registro da PA. Para cada eixo temático, foram elaboradas dez perguntas discursivas 
com suas respectivas respostas, as quais foram ilustradas em cartas coloridas numeradas de 1 a 10 .

Todas as fases do procedimento de coleta de dados foram realizadas por um dos autores e operacionalizadas da seguinte maneira:

Fase I (preliminar): os prontuários catalogados no Serviço de Arquivo Médico e Estatístico foram investigados antes da implementação do programa educativo, durante o mês de dezembro de 2015, utilizando-se o formulário QRPAP;

Fase II (implementação): nos meses de janeiro a maio de 2016, os profissionais de enfermagem foram recrutados e convidados a participar da intervenção educativa, por meio de uma carta convite que continha o Termo de Consentimento Livre e Esclarecido (TCLE) e informações detalhadas sobre a pesquisa, seus objetivos e sua importância para o desenvolvimento profissional. Em uma sala reservada, o questionário CTRPA-SHE era entregue a cada participante, imediatamente antes e imediatamente após a aplicação da intervenção. Um total de 38 profissionais foi submetido à aula expositiva-dialogada e ao jogo de tabuleiro, sendo os demais 63 submetidos apenas à aula expositiva-dialogada, por conta de sua carga de trabalho e da indisponibilidade de tempo para participar da intervenção. Grupos de 38 e 63 profissionais seriam suficientes para atingir um tamanho de efeito de 0,6 , quando comparadas as diferenças de desempenho entre os participantes recrutados ${ }^{(15)}$;

Fase III (exploratória): os prontuários catalogados no Serviço de Arquivo Médico e Estatístico foram investigados após a implementação do programa educativo, durante o mês de junho de 2016, utilizando-se o formulário QRPAP.

$O$ efeito do programa educativo foi avaliado pelo Modelo de Kirkpatrick ${ }^{(26)}$ nos níveis aprendizagem e comportamento. O nível aprendizagem foi aplicado para avaliar o conhecimento teórico dos profissionais de enfermagem, enquanto o nível comportamental avaliou a qualidade dos registros da PA nos períodos pré e pós-intervenção.

Os dados coletados foram armazenados em banco de dados, em dupla digitação, no programa Microsoft Excel 2010. Em seguida, foram processados no programa Statistical Package for the Social Science versão 20.0 para Windows e conduzidas as análises com o programa computacional $\mathrm{R}$ versão 3.2.2. Para as estimativas de tamanho de amostra, utilizou-se o pacote pwr ${ }^{(15) .}$

Análises descritivas foram realizadas para todas as variáveis, sendo descritas por frequências absolutas e relativas, medianas e quartis. Para comparar o desempenho dos profissionais de enfermagem quanto ao número de acertos no CTRPA-SHE, foram utilizados testes de Wilcoxon para dados pareados. Para comparar a qualidade dos registros de PA antes e após o programa educativo, foram utilizados os testes exato de Fisher e Qui-quadrado em medidas categóricas ou testes de Mann-Whitney para medidas numéricas ou ordinais em amostras independentes. Os testes de Wilcoxon para dados pareados e Mann-Whitney foram utilizados para comparar os profissionais que participaram das duas estratégias (aula e jogo) com aqueles que foram submetidos apenas à aula expositiva-dialogada. O nível de significância adotado para os testes foi de $a=0,05$.

O estudo foi submetido ao Comitê de Ética em Pesquisa da referida instituição hospitalar e aprovado sob o protocolo no 31962014.1.0000.0071/2015.

\section{Resultados}

Foram elegíveis ao estudo 664 prontuários, sendo 363 analisados no período pré-intervenção e 301 no período pós-intervenção. Ao considerar os dois períodos, três prontuários foram excluídos em razão de registros de abalo emocional e 19 por agitação psicomotora (Figura 1).
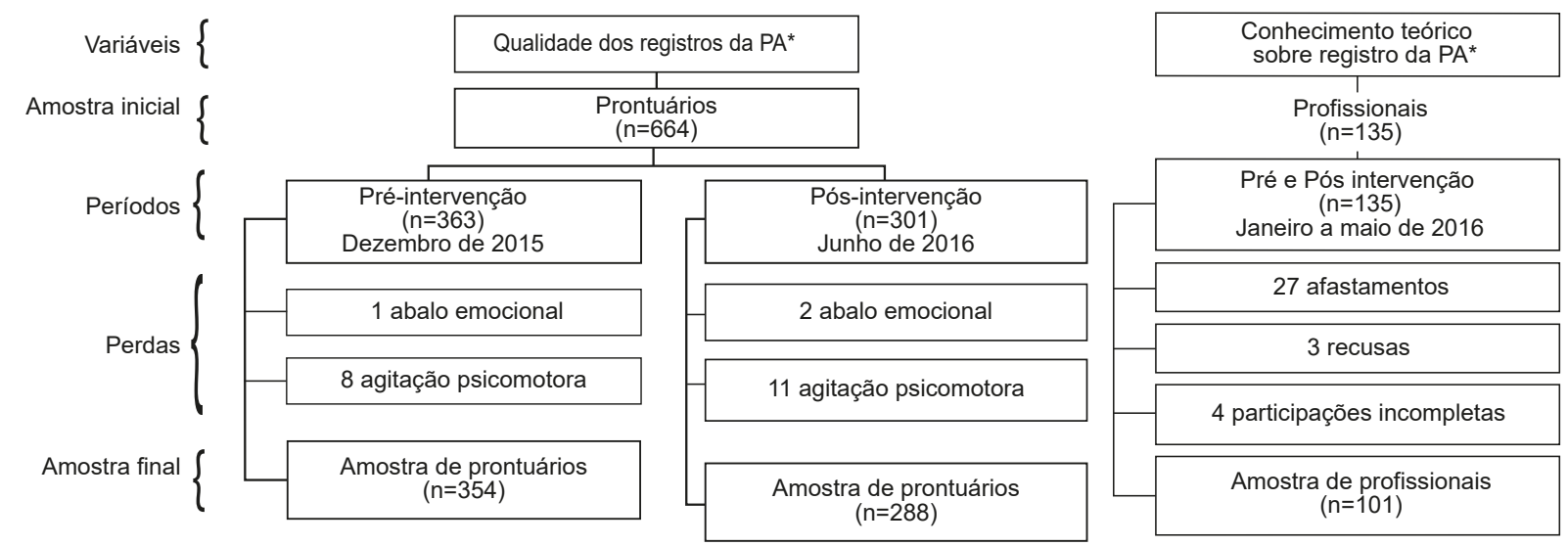

*PA $=$ pressão arterial

Figura 1 - Representação esquemática de inclusão e exclusão dos participantes no estudo conforme as variáveis de interesse e os períodos de intervenção. São Paulo, SP, Brasil, 2015-2016 
A amostra de profissionais foi composta por 135 participantes, dos quais 27 foram excluídos em razão dos afastamentos, três por recusa quanto à participação na pesquisa e mais quatro por não terem completado todas as etapas do estudo. Ao final, 642 prontuários e 101 profissionais de enfermagem compuseram as respectivas amostras (Figura 1 ).

A Figura 1 ilustra o fluxo de inclusão dos participantes no estudo conforme as variáveis de interesse e os períodos de intervenção.

A qualidade dos registros da PA foi analisada em 354 registros realizados no período pré-intervenção $(55,1 \%)$ e 288 no período pós-intervenção (44,9\%). No período pré-intervenção, dois prontuários $(0,6 \%)$ continham o registro da medida da $\mathrm{CB}$ e outros dois $(0,6 \%)$ possuíam o registro do membro em que foi realizada a medida da CB. Para o mesmo período, não foram encontrados registros sobre o tamanho do manguito e nem sobre a posição do paciente durante a realização do procedimento de medida da PA.

As análises dos dados referentes ao período pósintervenção mostraram aumento das frequências de registros para as variáveis "tamanho do manguito" $(24,7 \%)$, "membro em que foi medido a CB" $(29,2 \%)$ e "posição do paciente" (69,1\%). A Tabela 1 descreve e compara a qualidade dos registros da PA identificados nos prontuários analisados nos períodos pré e pós-intervenção.

Tabela 1 - Descrição e comparação da qualidade dos registros da pressão arterial identificados nos prontuários analisados nos períodos pré e pós-intervenção $(n=642)$. São Paulo, SP, Brasil, 2015-2016

\begin{tabular}{|c|c|c|c|}
\hline Qualidade dos registros da pressão arterial & $\begin{array}{l}\text { Período pré-intervenção } \\
(\mathrm{n}=354) \\
\mathrm{n}(\%)\end{array}$ & $\begin{array}{l}\text { Período pós-intervenção } \\
\qquad(\mathrm{n}=\mathbf{2 8 8}) \\
\mathrm{n}(\%)\end{array}$ & Valor $\mathbf{p}^{*}$ \\
\hline Registro da medida da $\mathrm{CB}^{+}$ & $2(0,6)$ & $1(0,3)$ & $>0,999$ \\
\hline Registro do tamanho do manguito & $0(0,0)$ & $71(24,7)$ & $<0,001$ \\
\hline Registro do membro em que foi medida a $\mathrm{CB}^{\dagger}$ & $2(0,6)$ & $84(29,2)$ & $<0,001$ \\
\hline Registro da posição do paciente & $0(0,0)$ & $199(69,1)$ & $<0,001$ \\
\hline
\end{tabular}

*Teste Exato de Fisher; ${ }^{+} \mathrm{CB}=$ circunferência braquial

Após a implementação do programa educativo, houve aumento significativo na proporção de registros sobre tamanho do manguito utilizado, membro em que foi medida a CB e a posição do paciente $(p<0,001)$.

A variável "registro da PA com unidade de medida do aparelho $(\mathrm{mmHg})$ " apresentou conformidade em $99,0 \%$ dos casos e não mostrou diferenças significativas entre os períodos pré e pós-intervenção $(p=0,900)$.

A amostra de trabalhadores de enfermagem foi composta por 42 enfermeiros, 58 técnicos de enfermagem e um auxiliar de enfermagem. As características desses profissionais que participaram do estudo e foram submetidos à avaliação do conhecimento teórico sobre registro da PA antes e após o programa educativo estão apresentadas na Tabela 2.

A frequência de respostas corretas obtida em cada questão do CTRPA-SHE foi analisada e está demostrada na Tabela 3.
Tabela 2 - Descrição das características profissionais dos participantes submetidos ao programa educativo $(n=101)$. São Paulo, SP, Brasil, 2016

\begin{tabular}{lcc}
\hline Características & $\mathbf{n}(\%)$ & \multicolumn{1}{c}{ Mediana [IIQ] } \\
\hline Idade (anos) & $34,00[29,00 ; 39,00]$ \\
Sexo Feminino & $75(74,3)$ & \\
Categoria profissional & & \\
$\quad$ Auxiliar & $1(1,0)$ & \\
$\quad$ Técnico & $58(57,4)$ & \\
$\quad$ Enfermeiro & $42(41,6)$ & \\
Tempo de formação profissional & & $9,00[4,92 ; 15,00]$ \\
(anos) & & $5,00[2,66 ; 10,00]$ \\
Tempo em que trabalha na & & \\
instituição (anos) & & \\
Turno de trabalho & & \\
$\quad$ Manhã & $37(36,6)$ & \\
Tarde & $36(35,6)$ & \\
$\quad$ Noite & $28(27,7)$ & \\
*IIQ = intervalo interquartil &
\end{tabular}

*IIQ = intervalo interquartil

Tabela 3 - Frequência de acertos dos profissionais de enfermagem em cada questão do questionário "Conhecimento Teórico sobre Registro da Pressão Arterial em Serviço Hospitalar de Emergência" nos períodos pré-intervenção e pósintervenção ( $n=101)$. São Paulo, SP, Brasil, 2016

\begin{tabular}{lc}
\hline Questões & \multicolumn{2}{c}{$\begin{array}{c}\text { Pré-intervenção } \\
\text { (n) }\end{array}$} & $\begin{array}{c}\text { Pós-intervenção } \\
\text { (n) }\end{array}$ & 82 \\
\hline $\begin{array}{l}\text { 1. Qual a importância do registro da PA* para o cuidado de enfermagem? } \\
\text { 2. Quais valores de PA* registrados no prontuário do paciente podem indicar um sinal de gravidade } \\
\text { hemodinâmica? }\end{array}$ & 93 \\
\hline
\end{tabular}


Tabela 3 - continuação

\begin{tabular}{|c|c|c|}
\hline Questões & $\begin{array}{l}\text { Pré-intervenção } \\
\text { (n) }\end{array}$ & $\begin{array}{l}\text { Pós-intervenção } \\
\text { (n) }\end{array}$ \\
\hline \multicolumn{3}{|l|}{ 3. Ao verificar valores de $\mathrm{PA}^{*}$ com alterações significativas, deve-se: } \\
\hline $\begin{array}{l}\text { a) Garantir que a medida da } \mathrm{PA}^{*} \text { tenha sido realizada com técnica correta, caso contrário, uma nova } \\
\text { medida deve ser feita. }\end{array}$ & 101 & 101 \\
\hline b) Comunicar os resultados obtidos ao paciente/acompanhante. & 82 & 91 \\
\hline c) Comunicar os valores obtidos para o enfermeiro/médico responsável pelo paciente. & 101 & 101 \\
\hline d) Registrar na anotação de enfermagem que o enfermeiro/médico foi comunicado e a conduta realizada. & 100 & 101 \\
\hline e) Comunicar à engenharia clínica a presença de falhas na leitura do aparelho. & 89 & 89 \\
\hline \multicolumn{3}{|l|}{$\begin{array}{l}\text { 4. Em relação às vantagens do uso de aparelhos automáticos para o registro da } \mathrm{PA}^{*} \text {, assinale verdadeiro ou } \\
\text { falso: }\end{array}$} \\
\hline a) Precisão dos resultados obtidos. & 79 & 95 \\
\hline b) Possibilidade de armazenamento dos valores da PA* no aparelho. & 90 & 99 \\
\hline c) Cálculo da pressão arterial média e valores descritos em até três dígitos. & 95 & 97 \\
\hline d) Realização de diversas medidas em um intervalo de tempo determinado pelo operador. & 91 & 95 \\
\hline e) Arredondamento de valores. & 89 & 90 \\
\hline 5. Devemos sempre registrar a posição do paciente durante a medida da PA*, porque: & 96 & 97 \\
\hline 6. Devemos sempre registrar a frequência cardíaca do paciente durante a medida da $\mathrm{PA}^{*}$, porque: & 46 & 79 \\
\hline $\begin{array}{l}\text { 7. Para selecionar e registrar, com praticidade, o tamanho do manguito que melhor se adequa ao braço do } \\
\text { paciente, deve-se: }\end{array}$ & 9 & 73 \\
\hline 8. A verificação da $\mathrm{PA}^{*}$ deve ser registrada: & 99 & 100 \\
\hline $\begin{array}{l}\text { 9. Para preencher o item da prescrição de enfermagem sobre frequência de verificação de sinais vitais, o } \\
\text { enfermeiro deve levar em consideração: }\end{array}$ & 99 & 99 \\
\hline \multicolumn{3}{|l|}{ 10. Em relação à frequência do registro da $\mathrm{PA}^{*}$, assinale verdadeiro ou falso: } \\
\hline $\begin{array}{l}\text { a) } \mathrm{APA}^{*} \text { de pacientes instáveis ou que estejam recebendo infusão de drogas vasoativas deve ser } \\
\text { registrada a cada } 15 \text { minutos, no máximo. }\end{array}$ & 94 & 101 \\
\hline b) $\mathrm{A} \mathrm{PA}^{*}$ de pacientes classificados como alto risco deve ser registrada a cada 2 horas, no máximo. & 78 & 100 \\
\hline c) A PA* de pacientes classificados como muito urgentes deve ser registrada a cada 4 horas, no máximo. & 87 & 100 \\
\hline d) A PA* de pacientes classificados como urgentes deve ser registrada a cada 6 horas, no máximo. & 96 & 101 \\
\hline e) A PA* de pacientes classificados como pouco urgentes ou não urgentes não deve ser registrada. & 88 & 90 \\
\hline $\begin{array}{l}\text { 11. Durante e após o procedimento de medida da } \mathrm{PA}^{*} \text { com aparelhos automáticos, deve-se anotar em } \\
\text { prontuário: }\end{array}$ & 63 & 96 \\
\hline
\end{tabular}

*PA $=$ pressão arterial

Houve melhora significativa na proporção de respostas corretas para a questão de número 1 que discorreu sobre "a importância do registro da pressão arterial para o cuidado em enfermagem" ( $p=0,006)$, de número 2 sobre "valores de pressão arterial que podem indicar sinal de gravidade hemodinâmica" ( $p=0,001)$, as alternativas a e b da questão de número 4 que abordaram a "precisão dos valores de PA obtidos com o uso de aparelhos automáticos" $(p=0,001)$ e "possibilidade de armazenamento de valores da PA nos aparelhos oscilométricos" $(p=0,027)$, a questão de número 6 sobre "registro da frequência cardíaca" $(p<0,001)$, de número 7 sobre "seleção do manguito" $(p<0,001)$, as alternativas $a, b$ e $c$ da questão de número 10 que abordavam a "frequência de registros da $\mathrm{PA}^{\prime}$ $(p=0,023, p<0,001$ e $p=0,002)$ e a questão de número 11 sobre "registro das etapas da medida da PA" $(p<0,001)$.

Ao considerar a contagem total de acertos obtidos no CTRPA-SHE, 80 profissionais de enfermagem (79,2\%) atingiram uma nota final maior após a implementação do programa educativo, $17(16,8 \%)$ mantiveram a mesma nota e quatro $(4,0 \%)$ apresentaram redução. A mediana dos pontos obtidos no período pré-intervenção foi de 19 acertos (10 quartil 18 e $3^{\circ}$ quartil 21 ), passando para 22 no período pós-intervenção (10 quartil 20 e $3^{\circ}$ quartil 23), de um total de 23 itens $(p<0,001)$. A Figura 2 mostra o total de acertos desses profissionais nos períodos pré-intervenção e pós-intervenção, quando aplicado o questionário CTRPA-SHE.

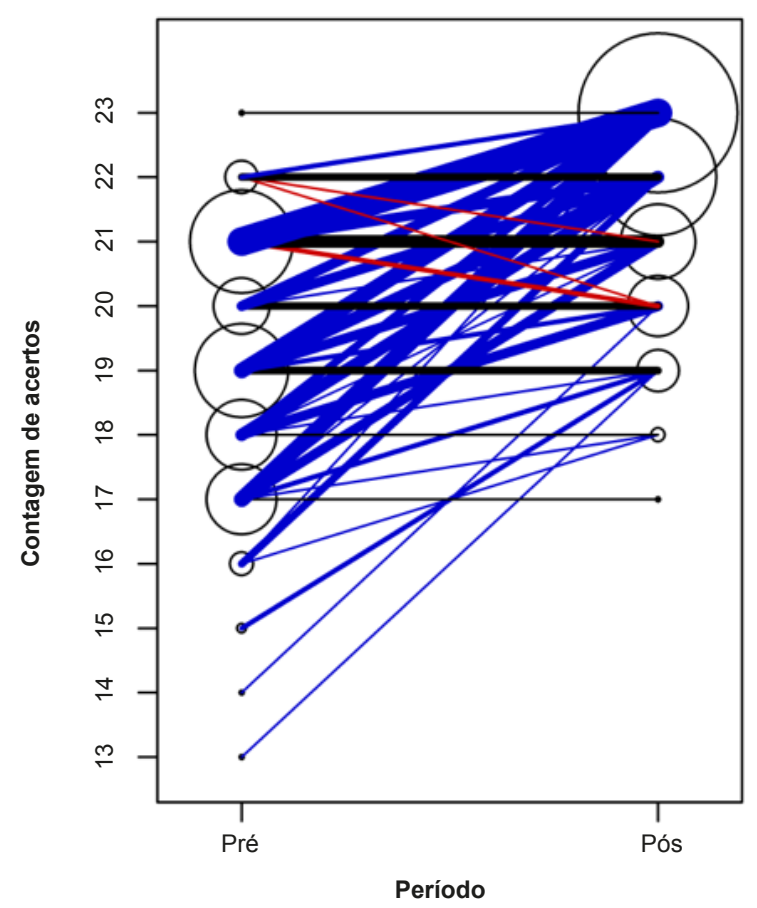

*Círculos maiores e linhas mais grossas indicam maior número de profissionais. A evolução de cada um está destacada em cores: azul se a nota aumentou, vermelho se a nota diminuiu e preto se manteve estável

Figura 2 - Total de acertos dos profissionais de enfermagem no questionário "Conhecimento Teórico sobre Registro da Pressão Arterial em Serviço Hospitalar de Emergência" nos períodos pré-intervenção e pós-intervenção $(n=101) *$. São Paulo, SP, Brasil, 2016 
Ao comparar o total de acertos entre as categorias profissionais, os resultados evidenciaram que não houve diferenças significativas no desempenho de auxiliares e técnicos de enfermagem ou de enfermeiros nos períodos pré-intervenção $(p=0,759)$ e pós-intervenção $(p=0,828)$. A mediana dos pontos obtidos por todas as categorias profissionais foi de 19 acertos no período pré-intervenção (10 quartil 18 e $3^{\circ}$ quartil 21 ) e 22 acertos no período pós-intervenção (10 quartil 20 e $3^{\circ}$ quartil 23 ).

Um total de 63 profissionais de enfermagem $(62,4 \%)$ participou da aula expositiva-dialogada, enquanto 38 profissionais $(37,6 \%)$ foram submetidos à aula e ao jogo de tabuleiro. A mediana de idade dos participantes do jogo de tabuleiro foi de 31 anos, sendo $84,2 \%$ do sexo feminino. Quanto à categoria profissional, 11 trabalhadores eram enfermeiros (28,9\%), 26 eram técnicos de enfermagem $(68,4 \%)$ e 1 era auxiliar de enfermagem $(2,6 \%)$

A mediana dos pontos obtidos pelos profissionais que participaram apenas da aula expositiva-dialogada foi de 19 acertos no período pré-intervenção (10 quartil 17 e $3^{\circ}$ quartil 21) e de 22 acertos no período pósintervenção ( $1^{\circ}$ quartil 20 e $3^{\circ}$ quartil 23). Já a mediana dos pontos obtidos pelos profissionais que participaram da aula expositiva-dialogada e do jogo de tabuleiro foi de 19 acertos no período pré-intervenção (10 quartil 18 e $3^{\circ}$ quartil 21) e de 22 acertos no período pósintervenção (10 quartil 21 e $3^{\circ}$ quartil 22). Os escores de acerto entre esses dois grupos não mostraram diferenças significativas nos períodos pré-intervenção $(p=0,412)$ e pós-intervenção $(p=0,273)$.

\section{Discussão}

Este estudo buscou avaliar o efeito de um programa educativo sobre o registro da PA para profissionais de enfermagem em relação ao conhecimento teórico e à qualidade desses registros. Até o presente momento, identificou-se que poucos são os estudos que se dedicaram a explorar essa temática e sua importância no contexto clínico e assistencial.

As etapas da medida indireta da PA compõem uma série de passos que subsidiam a realização do procedimento de forma correta e padronizada, conforme especificado por diretrizes nacionais e internacionais de $H A^{(1-2)}$. O registro de enfermagem referente às etapas desse procedimento já está elucidado na literatura, que consiste na documentação da medida da $\mathrm{CB}$, tamanho do manguito, membro utilizado, posição do paciente, horário de realização da medida, valores obtidos sem arredondamentos e frequência cardíaca ${ }^{(1-2)}$. O registro de todas essas etapas justifica-se pela necessidade de obtenção de valores fidedignos que representem o quadro clínico do paciente e contribuam para a definição do diagnóstico, da assertividade no tratamento, da monitorização e de seguimento das condições de saúde.

O estudo evidenciou melhora significativa na qualidade dos registros de PA quando comparadas as variáveis documentação da posição do paciente, tamanho do manguito e membro selecionado para a medida dos valores pressóricos, nos períodos pré e pós-intervenção. Tais resultados estão em concordância com outros achados da literatura consultada, os quais utilizaram métodos de pesquisa semelhantes e comprovaram a eficácia de estratégias educativas na promoção do conhecimento sobre o procedimento de medida indireta da PA e sua aplicabilidade na prática assistencial( ${ }^{(7-8)}$.

A escolha do manguito adequado, baseada na medida da CB do paciente, é necessária para a obtenção de valores fidedignos de $\mathrm{PA}^{(27-28)}$. Dessa forma, o manguito deve corresponder a $80 \%$ do comprimento e $40 \%$ da largura da $C B$, em uma razão comprimento/largura de $2: 1^{(2)}$. Embora manguitos de variados tamanhos sejam necessários para atender a uma gama de medidas de circunferências braquiais, os profissionais de saúde têm usado um tamanho padrão na medida da $\mathrm{PA}$, ora pela escolha inadequada, ora pela indisponibilidade dos manguitos nos serviços de saúde, ou ainda devido à falta de padronização de medidas pelos fabricantes(29).

Nos últimos anos, alternativas para diminuir o uso incorreto do manguito têm sido estudadas e discutidas pelas sociedades de especialistas em HA, com destaque para a orientação canadense, a qual defende a utilização de manguitos que delimitam o intervalo da $C B$ e indicam o tamanho adequado por meio de marcas ilustradas, gravadas no tecido que envolve a bolsa de borracha(2). Com base nessa nova prática, este estudo demonstrou melhora significativa na qualidade dos registros referentes ao tamanho do manguito selecionado. Acredita-se que a orientação quanto ao uso de manguitos ilustrados, em detrimento da realização da medida da $\mathrm{CB}$, possa trazer praticidade ao procedimento e contribuir com a diminuição de erros relacionados à utilização inadequada do equipamento.

A escolha do membro do paciente é considerada essencial para um cuidado individualizado, tendo em vista a necessidade de identificar aquele que apresenta maior valor pressórico e de torná-lo referência para a definição diagnóstica(1-2). Os resultados desta pesquisa foram positivos em relação ao registro do membro em que a PA foi verificada, entretanto, menos de $30 \%$ dos profissionais que participaram da intervenção educativa realizaram o registro na prática, fato que se assemelha às evidências encontradas em estudos anteriores ${ }^{(7)}$.

$O$ registro do membro utilizado no procedimento sinaliza aos demais profissionais quanto à possibilidade 
de situações que contraindiquem a medida no membro contralateral, tais como linfedemas, tromboses, enxertos, alterações isquêmicas, fístulas e utilização de dispositivos centrais ou periféricos ${ }^{(27)}$. Além disso, estudos já demonstraram a existência de diferenças nos valores de PAS e PAD em ambos os braços de pessoas mais velhas e de pacientes portadores de HA, diabetes mellitus, hipercolesterolemia e doença vascular periférica(30-31).

A posição do paciente durante a medida da PA é essencial para a interpretação dos valores obtidos, a definição do diagnóstico e a tomada de decisão nas condutas clínicas e cuidados assistenciais de enfermagem. Os valores da PAS e da PAD sofrem importantes alterações, se comparados às posições sentada, deitada e em pé, tendo em vista a forte influência dos fatores hidrostáticos e isométricos na regulação vascular sistêmica(32).

Um dos fatores de erro mais comuns nas etapas da medida indireta da PA está relacionado ao arredondamento de valores de PA e à preferência do observador em documentar determinados dígitos terminais, tais como zero, dois e quatro(33). Estudo demonstrou que o arredondamento dos valores da PA pode chegar a $70 \%$ em uma determinada amostra e está diretamente relacionado à baixa qualidade do cuidado e à falta de conhecimento teórico-prático do profissional da saúde para a realização do procedimento(7).

Os resultados desta pesquisa mostraram que, mesmo antes do período de aplicação do programa educativo, os profissionais de enfermagem realizaram 99\% dos registros de PA com unidades de medida compatíveis com a determinação do aparelho, em $\mathrm{mmHg}$. Tal fato pode estar associado à utilização de aparelhos oscilométricos digitais, os quais registram os valores de PA em até três dígitos e minimizam a preferência do observador aos dígitos terminais.

Em relação ao conhecimento teórico, os resultados mostraram que os profissionais de enfermagem apresentaram maiores escores de acerto no questionário CTRPA-SHE após a execução do programa educativo, com destaque para as questões que discorreram sobre o registro de PA em pacientes com instabilidade hemodinâmica, as vantagens do uso de aparelhos oscilométricos no registro da $\mathrm{PA}$, a frequência de registros da PA conforme o quadro clínico do paciente e o registro das etapas que compõem o procedimento de medida indireta da PA. Tais evidências sugerem o efeito positivo da intervenção conduzida neste estudo e da aplicabilidade de metodologias ativas de ensinoaprendizagem no contexto assistencial de enfermagem.

As metodologias ativas utilizam a problematização como facilitadora do processo de ensino-aprendizagem, uma vez que levam o estudante a relacionar a descoberta de fenômenos de interesse com seus conhecimentos prévios e com experiências do mundo real(34-35). Destacam-se como estratégias de ensino baseadas nessa metodologia a aula expositiva-dialogada, a discussão em grupo, a instrução individual, os jogos, a simulação e a dramatização, os quais promovem a participação ativa do aluno, a utilização de habilidades cognitivas, afetivas e psicomotoras, bem como desenvolvem o conhecimento teórico-prático, o pensamento crítico, o

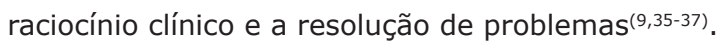

A aplicação de estratégias inovadoras tem demonstrado resultados efetivos na melhora do conhecimento teórico-prático sobre a medida indireta da $\mathrm{PA}^{(7,9)}$. Entretanto, várias são as evidências que comprovam a falta de qualidade dos registros em saúde e a existência de importantes lacunas no conhecimento de profissionais de enfermagem quanto à documentação do cuidado ao paciente e à aplicação prática das etapas do processo de enfermagem ${ }^{(38-39)}$.

Tanto o registro de enfermagem quanto a medida indireta da PA são temáticas abordadas constantemente nos cursos de graduação em enfermagem, uma vez que compõem o cotidiano da equipe de saúde e auxiliam o planejamento do cuidado prestado. Apesar disso, as competências adquiridas no ensino superior não têm sido suficientes para desenvolver o conhecimento e as habilidades necessárias às exigências do mercado de trabalho, o que torna o papel educativo das organizações de saúde uma peça-chave na formação profissional(40). 0 mesmo raciocínio aplica-se nos cursos para a formação dos trabalhadores de nível técnico dessa profissão.

Apesar do registro da PA ser considerado atividade simples e de fácil execução, acredita-se que a promoção do conhecimento por meio de programas de atualização e educação permanente em saúde contribua tanto para a capacitação técnica e científica dos profissionais, quanto para a segurança do paciente e a qualidade da assistência de enfermagem.

Este estudo colaborou com o desenvolvimento de um programa educativo inovador, que pôde ser avaliado por meio da aplicação de instrumentos de coleta de dados validados, capazes de mensurar o conhecimento teórico de profissionais de enfermagem sobre registro da PA e investigar a qualidade desses registros em prontuários de pacientes admitidos em um serviço hospitalar de emergência. As estratégias aqui demonstradas poderão ser adaptadas e aplicadas em outros serviços de saúde, para a promoção da educação em enfermagem.

Algumas limitações podem ser consideradas em relação à validade interna e externa dos resultados apresentados neste estudo, tais como a escolha por amostras não probabilísticas e as peculiaridades do local e da população selecionada. Sugerem-se o desenvolvimento 
de estudos experimentais com amostras mais representativas e a implementação de metodologias ativas de ensino-aprendizagem em outros contextos assistenciais e em serviços de atenção à saúde com diferentes níveis de investimento tecnológico e financeiro.

\section{Conclusão}

A implementação de um programa educativo para profissionais de enfermagem sobre registro da PA mostrou resultados positivos na promoção do conhecimento teórico e na qualidade dos registros de PA em um serviço hospitalar. A implementação de metodologias ativas de ensino-aprendizagem contribui para o desenvolvimento de habilidades técnicas, para o estabelecimento de práticas seguras e para a promoção do conhecimento profissional.

\section{Referências}

1. Malachias MVB, Souza WKSB, Plavnik FL, Rodrigues CIS, Brandão AANM, Bortolotto LA, et al. 7th Brazilian Guideline of Arterial Hypertension. Arq Bras Cardiol. [Internet]. 2016 Sept [cited 2018 May 10]; 107(3 Suppl 3):S1-83. Available from: http://publicacoes.cardiol.br/ 2014/ diretrizes/2016/05_HIPERTENSAO_ARTERIAL_ING LES.pdf.

2. Leung AA, Nerenberg K, Daskalopoulou SS, McBrien K, Zarnke KB, Dasgupta K, et al. Hypertension Canada's 2016 Canadian Hypertension Education Program Guidelines for Blood Pressure Measurement, Diagnosis, Assessment of Risk, Prevention, and Treatment of Hypertension. Can J Cardiol. [Internet]. 2016 May [cited 2018 May 10]; 32(5):569-88. Available from: https:// www.ncbi.nlm.nih.gov/pubmed/27118291.

3. Diniz SOS, Silva PS, Figueiredo NMA, Tonini T. Quality of nursing records: analytical reflections on its forms and contents. JNUOL. [Internet]. 2015 Sept 10 [cited 2018 May 17]; 9(10):9616-23. Available from: https://periodicos.ufpe. $\mathrm{br} /$ revistas/revistaenfermagem/article/ view/10908.

4. Teixeira CC, Boaventura RP, Souza ACS, Paranaguá TTDB, Bezerra ALQ, Bachion MM, et al. Vital signs measurement: an indicator of safe care delivered to elderly patients. Texto Contexto Enferm. [Internet]. 2015 Oct [cited 2018 May 27]; 24(4):1071-8. Available from: http://www.scielo.br/scielo.php?script=sci_arttext\&pid= S010407072015000401071\&lng=en\&nrm=iso\&tIng=en. 5. Mouro DL, Godoy SD, Veiga EV, Zandomenighi RC, Marchi-Alves LM. Practices adopted by nursing professionals for indirect measurement and recording of blood pressure. Rev Min Enferm. [Internet]. 2017 Jun [cited 2019 Jan 29]; 21:e-995. Available from: http:// www.revenf.bvs.br/scielo.php?script=sci_arttext\&pid $=$ S $141527622017000100205 \&$ lng =es\&nrm=iso $>$.

6. Melo GSM, Tibúrcio MP, Freitas CCS, Vasconcelos QLDAQ, Costa IKF, Torres GV. Semiotics and semiology of Nursing: evaluation of undergraduate students' knowledge on procedures. Rev Bras Enferm. [Internet]. 2017 MarApr [cited 2019 Feb 6]; 70(2):249-56. Available from: https://www.redalyc.org/html/2670/267050430005/.

7. Machado JP, Veiga EV, Ferreira PAC, Martins JCA, Daniel ACQG, Oliveira ADS, et al. Theoretical and practical knowledge of nursing professionals on indirect blood pressure measurement at a coronary care unit. Einstein. [Internet]. 2014 Sept 20 [cited 2018 Mar 26]; 12(3):330-5. Available from: http:// www.scielo.br/scielo.php?script=sci_arttext\&pid $=\mathrm{S} 167$ 945082014000300330\&lng=en\&nrm=iso\&tIng=en .

8. Sarmasoglu S, Dinç L, Elçin M. Using standardized patients in nursing education: effects on students' psychomotor skill development. Nurse Educ. [Internet]. 2016 Mar-Apr 2 [cited 2019 Feb 6]; 41(2):e1-e5. Available from: https://www.ncbi.nlm.nih.gov/pubmed/26102 639. 9. Andrade LZC, Freitas DT, Holanda GF, Silva VM, Lopes MVO, Araújo TL. Development and validation of an educational game: blood pressure measurement. Rev Enferm UERJ. [Internet]. 2012 Jan 29 [cited 2018 Mar 17]; 20(3):323-7. Available from: http://www.epublicacoes.uerj.br/index.php/enfermagemuerj/article/ view/1201/2877.

10. Gorbanev I, Agudelo-Londoño S, González RA, Cortes A, Pomares A, Delgadillo V, et al. A systematic review of serious games in medical education: quality of evidence and pedagogical strategy. Med Educ Online. [Internet]. 2018 Feb [cited 2019 Feb 1]; 23(1):1438718. Available from: https://www.tandfonline.com/doi/ full/10.1080/10872981.20 18.1438718.

11. AkI EA, Sackett KM, Erdley WS, Mustafa RA, Fiander M, Gabriel C, et al. Educational games for health professionals. Cochrane database of Systematic Reviews. [Internet]. 2013 Jan [cited 2019 Jan 30]; (3):1-11. Available from: https://www.cochranelibrary.com/cdsr/ doi/10.1002/14651858.CD006411.pub4/full.

12. Ellaway RH. A conceptual framework of game-informed principles for health professions education. Adv Simul (Lond). [Internet]. 2016 Jan [cited 2019 Jan 31]; 1(28):19. Available from: https://www.ncbi.nlm.nih.gov/pmc/ articles/PMC5806274/pdf/41077_2016_Article_30. pdf.

13. Dutra HS, Reis VND. Experimental and quasiexperimental study designs: definitions and challenges in nursing research. Rev UFPE. [Internet]. 2016 Jun [cited 2019 Jan 31]; 10(6):2230-41. Available from: http://www.revista.ufpe.br/revistaenfermagem/index. php/revista/article/vi ew/8847. 
14. Schweizer ML, Braun BI, Milstone AM. Research methods in healthcare epidemiology and antimicrobial stewardship-quasi-experimental designs. Infection control \& hospital epidemiology. [Internet]. 2016 Oct [cited 2019 Jan 31]; 37(10):1135-40. Available from: https://jhu.pure.elsevier.com/en/publications/ research-methods-in-healthcare-epidemiology-andantimicrobial-ste-3.

15. Champely S, Dalgaard P, Gill J, Weibelzahl S, Anandkumar A, Ford C, et al. Pwr: Basic Functions for Power Analysis. R package version 1.2-2. [software]. 2018 [cited 2019 Feb 02]. Available from: https:// CRAN.R-project.org/package $=$ pwr.

16. Almeida TDCF, Lamas JLT. Nurses of adult intensive care unit: evaluation about direct and indirect blood pressure measurement. Rev Esc Enferm USP. [Internet]. 2013 Apr [cited 2019 Feb 1]; 47(2):369-76. Available from: http:// www.scielo.br/scielo.php?script= sci_arttext\&pid=S008062342013000200014\&lng=en\&nrm=iso\&tIng=en.

17. Moreira MAD, Bernardino Júnior R. Analysis of health professionals theoretical/practical knowledge on indirect measurement of blood pressure. Biosci J. [Internet]. 2013 Feb [cited 2019 Feb 1]; 29(1):247-54. Available from: http://www.seer.ufu.br/index.php/ biosciencejournal/ article/view/9153/11994.

18. Tibúrcio MP, Melo GSM, Balduíno LSC, Freitas CCS, Costa IKF, Torres GV .Content validation of an instrument to assess the knowledge about the measurement of blood pressure. J Res Fundam Care. [Internet]. 2015 Abr [cited 2019 Feb 1]; 7(2):11. Available from: http:// www.seer.unirio.br/index.php/cuidadofundamental/ article/view/3585>.

19. DISABKIDS. The DISABKIDS Project [Internet]. Alemania: Pabs Science Publishers; 2012. [update 2018; cited 2019 Feb 6]. Available from: https://www. disabkids.org/.

20. Trevisol DJ, Silva A, Souza F, Zapelini CM. Development and validation of a polycystic liver disease complaint-specific assessment (POLCA): use of the Delphi technique for content validation. J Hepatol. [Internet]. 2015 Nov [cited 2019 Feb 1]; 62(4):988. Available from: https://www.sciencedirect.com/science/ article/abs/pii/S0168827814004541.

21. Rutherford-Hemming T. Determining content validity and reporting a content validity index for simulation scenarios. Nurs Educ Perspect. [Internet]. 2015 Sept [cited 2019 Feb 2]; 36(6):389-93. Available from: https://insights.ovid.com/crossref? an=00006199-200709000-00010.

22. Mancia G, Fagard R, Narkiewicz K, Redón J, Zanchetti A, Böhm M, et al. 2013 ESH/ESC Guidelines for the management of arterial hypertension: The Task Force for the management of arterial hypertension of the European
Society of Hypertension (ESH) and of the European Society of Cardiology (ESC). J Hypertens. [Internet]. 2013 Jul 21[cited 2018 Mar 12]; 31(7):1281-357. Available from: https://academic.oup.com/eurheartj/ article/34/28/2159/451304.

23. Daskalopoulou SS, Rabi DM, Zarnke KB, Dasgupta K, Nerenberg K, Cloutier L, et al. The 2015 Canadian Hypertension Education Program Recommendations for Blood Pressure Measurement, Diagnosis, Assessment of Risk, Prevention, and Treatment of Hypertension. Can ] Cardiol. [Internet]. 2015 May [cited 2018 Mar 14]; 31(5):549-68. Available from: https://www.ncbi.nlm. nih.gov/pubmed/25936483.

24. Berbel NAN. Active methodologies and the nurturing of students' autonomy. Semina: Ciências Sociais e Humanas. [Internet]. 2011 Jan [cited 2019 Feb 4]; 32(1):25-40. Available from: http://www.uel.br/revistas/ uel/index.php/seminasoc/article/view/10326/10999.

25. Freire P. Pedagogia da Autonomia: saberes necessários à prática educativa. 51st ed. Rio de Janeiro: Paz e terra; 2015.

26. Dorri S, Akbari M, Sedeh MD. Kirkpatrick evaluation model for in-service training on cardiopulmonary resuscitation. Iran J Nurs Midwifery Res. [Internet]. 2016 Sept-Oct [cited 2018 May 18]; 21(5):493-97. Available from: https://www.ncbi.nlm.nih.gov/pmc/ articles/PMC5114794/.

27. American Association of Critical-Care Nurses. Obtaining Accurate Noninvasive Blood Pressure Measurements in Adults. Crit Care Nurse. [Internet]. 2016 Jun [cited 2018 May 13]; 36(3):e12-e16. Available from: http://ccn.aacnjournals.org/content/36/3/e12.full. 28. Badeli $H$, Assadi F. Strategies to reduce pitfalls in measuring blood pressure. Int J Prev Med. [Internet]. 2014 Mar [cited 2018 Jun 19]; 5(Suppl 1):S17-20. Available from: https://www.ncbi.nlm.nih.gov/pmc/ articles/PMC3990924/.

29. Maia KAP, Malachias MVB, Paiva IV, Mariano RM, Paiva RV. Inadequacies of Sphygmomanometers Used in Emergency Care Services in a Large Capital City in Brazil. Int J Cardiovasc Sci. [Internet]. 2017 Apr [cited 2019 Feb 6]; 30(2):100-8. Available from: http://www.scielo. br/scielo.php?script=sci_arttext\&pid=S235956472017 000200100\&lng=en. http://dx.doi.org/10.5935/23594802.20170028.

30. Rosenberger J, McCrudden S, McCullough C, Wang L, Kime J, Albert NM. Factors associated with interarm blood pressure differences in patients admitted to critical care units. Heart Lung. [Internet]. 2018 Mar [cited 2019 Feb 2]; 47(2):100-6. Available from: https://www.sciencedirect.com/science/article/pii/ S0147956317302297. 
31. Weinberg I, Gona P, O'Donnell CJ, JaffMR, Murabito JM. The systolic blood pressure difference between arms and cardiovascular disease in the Framingham Heart Study. Am J Med. [Internet]. 2014 Mar [cited 2019 Feb 5]; 127(3):209-15. Available from: https://www.amjmed. com/article/S0002-9343(13)00972-8/fulltext.

32. Lacruz ME, Kluttig A, Kuss O, Tiller D, Medenwald D, Nuding $S$, et al. Short-term blood pressure variability variation between arm side, body position and successive measurements: a population-based cohort study. BMC Cardiovasc Disord. [Internet]. 2017 Jan [cited 2019 Feb 5]; 17(1):31. Available from: https://www.ncbi.nlm.nih.gov/ pmc/articles/PMC5241970/.

33. Ayodele OE, Sanya EO, Okunola OO, Akintunde AA. End digit preference in blood pressure measurement in a hypertension specialty clinic in southwest Nigeria. Cardiovasc ] Afr. [Internet]. 2012 Mar [cited 2019 Feb 5]; 23(2):85-9. Available from: https://www.ncbi.nlm.nih.gov/ pmc/articles/PMC3721825/.

34. Lacerda FCB, Santos LM. Completeness in top level training: methodology learning live. Avaliação. (Campinas) [Internet]. 2018 Sept [cited 2019 Fev 5]; 23(3):611-27. Available from: <http://www.scielo.br/ scielo.php?script=sci_arttext\&pid=S14144077201 $8000300611 \&$ Ing $=$ en\&nrm $=$ iso $>$.

35. Côrtes CT, Oliveira SMJV, Santos RCS, Francisco AA, Riesco MLG, Shimoda GT. Implementation of evidencebased practices in normal delivery care. Rev. LatinoAm. Enfermagem. [Internet]. 2018 [cited 2019 Mar 12]; 26:e-2988. Available from: http://www. scielo.br/scielo.php?script=sci_arttext\&pid=S0104$11692018000100304 \&$ Ing=pt.

36. Gurgel SDS, Taveira GP, Matias EO, Pinheiro PNDC, Vieira NFC, LimaFET. Educational games: didacticresources utilized at teaching health education classes. REME Rev Min Enferm. [Internet]. 2017 Out [cited 2019 Feb 5]; 2:e-1016. Available from: http://www.reme.org.br/ artigo/detalhes/1152.

37. Teles LMR, Américo CF, Oriá MOB, Vasconcelos CTM, Brüggemann OM, Damasceno AKC. Efficacy of an educational manual for childbirth companions: pilot study of a randomized clinical trial. Rev. Latino-Am. Enfermagem. [Internet]. 2018 [cited 2019 Mar 12]; 26:e-2996. Available from: http://www.scielo.br/scielo.php?script=sci_ arttext\&pid=S010 411692018000100306\&lng=pt.

38. De Groot K, Triemstra M, Paans W, Francke AL. Quality criteria, instruments, and requirements for nursing documentation: a systematic review of systematic reviews. J Adv Nurs. [Internet]. 2019 [cited 2019 Feb 5]; 00:1-15. Available from: https://onlinelibrary. wiley.com/ doi/full/10.1111/jan.13919.

39. Rajkovič U, Kapun MM, Dinevski D, Prijatelj V, Zaletel M, Šušteršič $O$. The Status of Nursing Documentation in Slovenia: a Survey. J Med Syst. [Internet]. 2016 Sep [cited 2019 Feb 5]; 40(9):198. Available from: https://link. springer.com/article/10.1007\%2Fs10916-016-0546-x.

40. Ortega MDCB, Cecagno D, Llor MAS, de Siqueira $\mathrm{HCH}$, Montesinos MJL, Soler LM. Academic training of nursing professionals and its relevance to the Workplace. Rev. Latino-Am. Enfermagem. [Internet]. 2015 May-Jun [cited 2019 Feb 5]; 23(3):404-10. Available from: https://www.revistas.usp. br/rlae/article/view/102729.
Copyright $\odot 2019$ Revista Latino-Americana de Enfermagem Este é um artigo de acesso aberto distribuído sob os termos da Licença Creative Commons CC BY.

Esta licença permite que outros distribuam, remixem, adaptem e criem a partir do seu trabalho, mesmo para fins comerciais, desde que Ihe atribuam o devido crédito pela criação original. É a licença mais flexível de todas as licenças disponíveis. É recomendada para maximizar a disseminação e uso dos materiais licenciados. 